\title{
Estimation of the parameters in two linear models with some of the identical parameter vectors under the Pitman's closeness criterion
}

Jibo Wu*

Chongqing Key Laboratory of Complex Data Analysis and Artificial Intelligence, Chongqing University of Arts and Sciences, Chongqing, China.

\begin{abstract}
Two normal linear models for some of the identical parameters are discussed in this article. Many authors have studied the properties of estimators in two normal linear models for some of the identical parameters using mean squared error and mean squared error matrix criteria. In this article we give comparison between the estimators in two normal linear models with some identical parameters under Pitman's closeness criterion with known variances. It is noted that the estimators in two linear models with some identical parameters with known variances are superior over the estimators in single linear models with some identical parameters with known variances. Finally, the simulation is carried out to show that the theoretical results which we obtained in this study are aligned with different simulation conditions. The simulation results agree with our theoretical results.
\end{abstract}

Keywords: Best linear unbiased estimator, common parameters, mean squared error, Pitman's closeness criterion, two normal linear models.

\section{INTRODUCTION}

Consider the following system $(\mathrm{H})$, which consists of two linear models:

$$
\begin{aligned}
& y_{1}=X_{1} \beta+Z_{1} \beta_{1}+\varepsilon_{1} \\
& y_{2}=X_{2} \beta+Z_{2} \beta_{2}+\varepsilon_{2}
\end{aligned}
$$

where $y_{i}$ shows a $n_{i} \times 1$ vector of observations, ( $i=1,2), X_{i}$ and $Z_{i}$ present $n_{i} \times p$ and $n_{i} \times t_{i}$ full rank matrices satisfying $\operatorname{rank}\left(X_{i}, Z_{i}\right)=\operatorname{rank}\left(X_{i}\right)+\operatorname{rank}$
$\left(Z_{i}\right)$ for rank(.) stands for the rank of a matrix. $\beta$ and $\beta_{i}$ show $n \times 1$ and $t_{i} \times 1$ unknown parameters, $\varepsilon_{i}$ present $n_{i} \times 1$ random vector supposed to satisfy a multivariate normal distribution with mean 0 and variance covariance matrix $\sigma_{i} I, \sigma_{i}$ are known parameters, and $\varepsilon_{1}$ is independent of $\varepsilon_{2}$.

Define $Q_{i}=I_{n i}-Z_{i}\left(Z_{i}^{\prime} Z_{i}\right)^{-1} Z_{i}^{\prime}, T_{i}=\left(Z_{i}^{\prime} Z_{i}\right)^{-1} Z_{i}^{\prime} X_{i}$ and $r=\frac{\sigma_{1}}{\sigma_{2}}$, then by Liu (1996), we have the following results:

(1) For the single equation (1), the best linear unbiased estimator (BLUE) of $\beta$ and $\beta_{1}$ are defined by,

$$
\begin{aligned}
& \hat{\beta}=\left(X_{1}^{\prime} Q_{1} X_{1}\right)^{-1} X_{1}^{\prime} Q_{1} y_{1} \\
& \hat{\beta}_{1}=\left(Z_{1}^{\prime} Z_{1}\right)^{-1} Z_{1}^{\prime} y_{1}-T_{1} \hat{\beta}
\end{aligned}
$$

(2) For the single equation (2), the BLUE of $\beta$ and $\beta_{2}$ are presented by,

$$
\begin{gathered}
\tilde{\beta}=\left(X_{2}^{\prime} Q_{2} X_{2}\right)^{-1} X_{2}^{\prime} Q_{2} y_{2} \\
\tilde{\beta}_{2}=\left(Z_{2}^{\prime} Z_{2}\right)^{-1} Z_{2}^{\prime} y_{2}-T_{2} \tilde{\beta}
\end{gathered}
$$

(3) For the system (H), the BLUE of $\beta, \beta_{1}$ and $\beta_{2}$ are presented by,

$$
\beta^{*}(r)=\left(X_{1}^{\prime} Q_{1} X_{1}+r X_{2}^{\prime} Q_{2} X_{2}\right)^{-1}\left(X_{1}^{\prime} Q_{1} y_{1}+r X_{2}^{\prime} Q_{2} y_{2}\right)
$$

\footnotetext{
*linfen52@126.com; (D https://orcid.org/0000-0001-6233-6704
} 


$$
\begin{aligned}
& \beta_{1}^{*}=\left(Z_{1}^{\prime} Z_{1}\right)^{-1} Z_{1}^{\prime} y_{1}-T_{1} \beta^{*}(r) \\
& \beta_{2}^{*}=\left(Z_{2}^{\prime} Z_{2}\right)^{-1} Z_{2}^{\prime} y_{2}-T_{2} \beta^{*}(r)
\end{aligned}
$$

In this article, we only study the estimation of the parameter $\beta$. Liu (1996) has presented the comparison between the estimators $\hat{\beta}, \tilde{\beta}$ and $\beta^{*}(r)$ in the mean squared error criterion when $\sigma_{i}$ is known. He also gave an estimator when $\sigma_{i}$ is unknown, and discussed the statistical properties of the estimators $\hat{\beta}, \tilde{\beta}$ and $\beta^{*}(r)$. Ma and Wang (2009) also discussed the estimators $\hat{\beta}, \tilde{\beta}$ and $\beta^{*}(r)$ in the mean squared error criterion.

Pitman closeness (PC) criterion was presented by Pitman (1937). Due to computation difficulty, it was used very rarely. [Since Rao (1981) and Keating et al. (1993) have organised a meeting to discuss the PC criterion, then] PC criterion has obtained considerable attention as an important approach to compare the estimators. Since a meeting held by Rao and kentry In literature, many authors have compared estimators using PC criterion. Wang and Yang (1994) used the PC criterion to compare two linear estimators in linear regression model and Reif (2006) used PC criterion to compare general pre-test estimators with some regression estimators. Yang et al. (2010) used it to compare two unified biased estimators in the linear regression model. Ahmadi and Balakrishnan $(2009 ; 2010)$ used it to compare some order statistics, while Jozani (2014) studied the PC using the balanced loss function. Li et al. (2012) used the PC criterion to compare the $r-k$ class estimator with the ordinary least squares estimator in linear regression model. $\mathrm{Wu}$ (2014) compared the modified $r-k$ class estimator with the ordinary least squares estimator in linear regression model under PC criterion. Wu (2017) also compared the estimators under the PC criterion.

Liu (1996) gave the comparison between the estimators $\hat{\beta}, \tilde{\beta}$ and $\beta^{*}(r)$ in the mean squared error criterion when $\sigma_{i}$ is known, however the comparison between the estimators $\hat{\beta}, \tilde{\beta}$ and $\beta^{*}(r)$ in the PC criterion was not done. In this study, we provide the comparison among the $\hat{\beta}, \tilde{\beta}$ and $\beta^{*}(r)$ in the PC criterion when $\sigma_{i}$ is known.

\section{RESULTS}

First, we present some definitions which are needed to prove some of the obtained results.

Definition 2.1. Suppose that $\hat{\theta}_{1}$ and $\hat{\theta}_{2}$ are two estimators of the unknown p-dimensional vector $\theta$. The
PC of $\hat{\theta}_{1}$ relative to $\hat{\theta}_{2}$ to estimate $\theta$ under a loss function $L(., \theta)$ is denoted as $P C\left(\hat{\theta}_{1}, \hat{\theta}_{2}, \theta\right)=P_{r}\left(\hat{\theta}_{1}, \hat{\theta}_{2}, \theta\right)=$ $P_{r}\left(\Delta\left(\hat{\theta}_{1}, \hat{\theta}_{2}\right) \geq 0\right)$, where

$\Delta\left(\hat{\theta}_{1}, \hat{\theta}_{2}\right)=L\left(\hat{\theta}_{2}, \theta\right)-L\left(\hat{\theta}_{1}, \theta\right)$

In this article, we study the quadratic loss function $L(\hat{\theta}, \theta)=(\hat{\theta}-\theta)^{\prime} U(\hat{\theta}-\theta)$ with a positive definite matrix, u.

Definition 2.2. $\hat{\theta}_{1}$ is said to be better than $\hat{\theta}_{2}$ for all $\theta \in \Theta$ in PC [under the loss function $L(., \theta)$, with some parameter space $\Theta]$, if

$P C\left(\hat{\theta}_{1}, \hat{\theta}_{2}, \theta\right)=P_{r}\left(\hat{\theta}_{1}, \hat{\theta}_{2}, \theta\right)=P_{r}\left(\Delta\left(\hat{\theta}_{1}, \hat{\theta}_{2}\right) \geq 0\right) \geq \frac{1}{2}$, for all $\theta \in \Theta$

\section{Comparison of the estimator $\hat{\beta}$ and the estimator $\beta^{*}(r)$ under the PC criterion}

Now we give the comparison of the estimator $\beta^{*}(r)$ given in equation (3) and the estimator $\beta^{*}(r)$ given in equation (7) under the PC criterion.

Theorem 2.1. Let $\sigma_{1}, \sigma_{2}$ are known and $\sigma_{2} \leq 2 \sigma_{1}$, then the estimator $\beta^{*}(r)$ given in equation (7) is superior over the estimator $\hat{\beta}$ given in equation (3) under the PC criterion.

Proof. Since matrices $X_{1}^{\prime} Q_{1} X_{1}$ and $X_{2}^{\prime} Q_{2} X_{2}$ are positive definite matrices (Liu, 1996), they can be diagonalised simultaneously, that is to say, there exists a reversible matrix $H$ such that,

$H^{\prime} X_{1}^{\prime} Q_{1} X_{1} H=I_{p}$

and

$H^{\prime} X_{2}^{\prime} Q_{2} X_{2} H=\operatorname{diag}\left(m_{1}, \ldots, m_{p}\right)=M_{p}$

for $m_{1}, \ldots, m_{p}$ present the positive eigenvalues of matrix $X_{2}^{\prime} Q_{2} X_{2}\left(X_{1}^{\prime} Q_{1} X_{1}\right)^{-1}$. Define:

$H^{\prime} X_{1}^{\prime} Q_{1} \varepsilon_{1}=\nu_{1}$

$r M_{p}^{-1 / 2} H^{\prime} X_{2}^{\prime} Q_{2} \varepsilon_{2}=\tau_{1}$

with $\nu_{1}$ and $\tau_{1}$ stand for p-dimension column random vector. It is easy to calculate that $\nu_{1} \sim N\left(0, \sigma_{1} I_{p}\right)$ and $\tau_{1} \sim N\left(0, \sigma_{1} I_{p}\right)$, and $\nu_{1}$ is independent of $\tau_{1}$. Choose $U=X_{1}^{\prime} Q_{1} X_{1}$. Since $Q_{i} Z_{i}=0, i=1,2$, by definition 2.1, we have, 


$$
\begin{aligned}
L(\hat{\beta}, \beta)= & (\hat{\beta}-\beta)^{\prime} X_{1}^{\prime} Q_{1} X_{1}(\hat{\beta}-\beta) \\
= & \left(\left(X_{1}^{\prime} Q_{1} X_{1}\right)^{-1} X_{1}^{\prime} Q_{1} y_{1}-\beta\right)^{\prime} X_{1}^{\prime} Q_{1} X_{1}\left(\left(X_{1}^{\prime} Q_{1} X_{1}\right)^{-1} X_{1}^{\prime} Q_{1} y_{1}-\beta\right) \\
= & \left(H^{\prime} X_{1}^{\prime} Q_{1} \varepsilon_{1}\right)^{\prime}\left(H^{\prime} X_{1}^{\prime} Q_{1} \varepsilon_{1}\right)=\nu_{1}^{\prime} \nu_{1} \\
L\left(\beta^{*}(r), \beta\right)= & \left(\beta^{*}(r)-\beta\right)^{\prime} X_{1}^{\prime} Q_{1} X_{1}\left(\beta^{*}(r)-\beta\right) \\
= & \left(\left(X_{1}^{\prime} Q_{1} X_{1}+r X_{2}^{\prime} Q_{2} X_{2}\right)^{-1}\left(X_{1}^{\prime} Q_{1} y_{1}+r X_{2}^{\prime} Q_{2} y_{2}\right)-\beta\right)^{\prime} X_{1}^{\prime} Q_{1} X_{1} \\
& \times\left(\left(X_{1}^{\prime} Q_{1} X_{1}+r X_{2}^{\prime} Q_{2} X_{2}\right)^{-1}\left(X_{1}^{\prime} Q_{1} y_{1}+r X_{2}^{\prime} Q_{2} y_{2}\right)-\beta\right) \\
= & \left(H^{\prime} X_{1}^{\prime} Q_{1} \varepsilon_{1}+r H^{\prime} X_{2}^{\prime} Q_{2} \varepsilon_{2}\right)^{\prime}\left(I_{p}+r M_{p}\right)^{-2} \\
& \times\left(H^{\prime} X_{1}^{\prime} Q_{1} \varepsilon_{1}+r H^{\prime} X_{2}^{\prime} Q_{2} \varepsilon_{2}\right) \\
= & \left(\nu_{1}+M_{p}^{1 / 2} \tau_{1}\right)^{\prime}\left(I_{p}+r M_{p}\right)^{-2}\left(\nu_{1}+M_{p}^{1 / 2} \tau_{1}\right) \\
= & \nu_{1}^{\prime}\left(I_{p}+r M_{p}\right)^{-2} \nu_{1}+\nu_{1}^{\prime} M_{p}^{1 / 2}\left(I_{p}+r M_{p}\right)^{-2} \tau_{1} \\
& +\tau_{1}^{\prime} M_{p}^{1 / 2}\left(I_{p}+r M_{p}\right)^{-2} \nu_{1}+\tau_{1}^{\prime} M_{p}\left(I_{p}+r M_{p}\right)^{-2} \tau_{1}
\end{aligned}
$$

Then we have,

$$
\begin{aligned}
P C\left(\beta^{*}(r), \hat{\beta}, \beta\right)= & P_{r}\left(L\left(\beta^{*}(r), \beta\right) \leq L(\hat{\beta}, \beta)\right) \\
= & P_{r}\left\{\nu_{1}^{\prime}\left(I_{p}+r M_{p}\right)^{-2} \nu_{1}+\nu_{1}^{\prime} M_{p}^{1 / 2}\left(I_{p}+r M_{p}\right)^{-2} \tau_{1}\right. \\
& \left.+\tau_{1}^{\prime} M_{p}^{1 / 2}\left(I_{p}+r M_{p}\right)^{-2} \nu_{1}+\tau_{1}^{\prime} M_{p}\left(I_{p}+r M_{p}\right)^{-2} \tau_{1} \leq \nu_{1}^{\prime} \nu_{1}\right\} \\
= & P_{r}\left\{\nu_{1}^{\prime}\left(\left(I_{p}+r M_{p}\right)^{-2}-I\right) \nu_{1}+\nu_{1}^{\prime} M_{p}^{1 / 2}\left(I_{p}+r M_{p}\right)^{-2} \tau_{1}\right. \\
& \left.+\tau_{1}^{\prime} M_{p}^{1 / 2}\left(I_{p}+r M_{p}\right)^{-2} \nu_{1}+\tau_{1}^{\prime} M_{p}\left(I_{p}+r M_{p}\right)^{-2} \tau_{1} \leq 0\right\} \\
= & P_{r}\left\{-\nu_{1}^{\prime}\left(I_{p}+r M_{p}\right)^{-2}\left(2 r M_{p}+r^{2} M_{p}^{2}\right) \nu_{1}+\nu_{1}^{\prime} M_{p}^{1 / 2}\left(I_{p}+r M_{p}\right)^{-2} \tau_{1}\right. \\
& \left.+\tau_{1}^{\prime} M_{p}^{1 / 2}\left(I_{p}+r M_{p}\right)^{-2} \nu_{1}+\tau_{1}^{\prime} M_{p}\left(I_{p}+r M_{p}\right)^{-2} \tau_{1} \leq 0\right\} \\
\geq & P_{r}\left\{-2 r \nu_{1}^{\prime}\left(I_{p}+r M_{p}\right)^{-2} M_{p} \nu_{1}+\nu_{1}^{\prime} M_{p}^{1 / 2}\left(I_{p}+r M_{p}\right)^{-2} \tau_{1}\right. \\
& \left.+\tau_{1}^{\prime} M_{p}^{1 / 2}\left(I_{p}+r M_{p}\right)^{-2} \nu_{1}+\tau_{1}^{\prime} M_{p}\left(I_{p}+r M_{p}\right)^{-2} \tau_{1} \leq 0\right\}
\end{aligned}
$$

Since $r \geq \frac{1}{2}$, we have $2 r \geq 1$, that is $\sigma_{2} \leq 2 \sigma_{1}$. Then we obtain the following:

$P C\left(\beta^{*}(r), \hat{\beta}, \beta\right) \geq$

$$
\begin{aligned}
& P_{r}\left\{-\nu_{1}^{\prime}\left(I_{p}+r M_{p}\right)^{-2} M_{p} \nu_{1}+\nu_{1}^{\prime} M_{p}^{1 / 2}\left(I_{p}+r M_{p}\right)^{-2} \tau_{1}\right. \\
& \left.+\tau_{1}^{\prime} M_{p}^{1 / 2}\left(I_{p}+r M_{p}\right)^{-2} \nu_{1}+\tau_{1}^{\prime} M_{p}\left(I_{p}+r M_{p}\right)^{-2} \tau_{1} \leq 0\right\}
\end{aligned}
$$

Now we compute,

$P C\left(\beta^{*}(1), \hat{\beta}, \beta\right) \geq$

$$
\begin{aligned}
& P_{r}\left\{-\nu_{1}^{\prime}\left(I_{p}+r M_{p}\right)^{-2} M_{p} \nu_{1}+\nu_{1}^{\prime} M_{p}^{1 / 2}\left(I_{p}+r M_{p}\right)^{-2} \tau_{1}\right. \\
& \left.+\tau_{1}^{\prime} M_{p}^{1 / 2}\left(I_{p}+r M_{p}\right)^{-2} \nu_{1}+\tau_{1}^{\prime} M_{p}\left(I_{p}+r M_{p}\right)^{-2} \tau_{1} \leq 0\right\} .
\end{aligned}
$$

Denote $\eta_{1}=\frac{1}{\sigma_{1}^{-1 / 2}} \nu_{1}, \eta_{2}=\frac{1}{\sigma_{1}^{-1 / 2}} \tau_{1}$, then $\eta_{1} \sim N(0, I)$ and $\eta_{2} \sim N(0, I)$. Therefore, equation (20) can be reduced to:
$P C\left(\beta^{*}(1), \hat{\beta}, \beta\right) \geq$

$$
\begin{aligned}
& P_{r}\left\{-\eta_{1}^{\prime}\left(I_{p}+r M_{p}\right)^{-2} M_{p} \eta_{1}+\eta_{1}^{\prime} M_{p}^{1 / 2}\left(I_{p}+r M_{p}\right)^{-2} \eta_{2}\right. \\
& \left.+\eta_{2}^{\prime} M_{p}^{1 / 2}\left(I_{p}+r M_{p}\right)^{-2} \eta_{1}+\eta_{2}^{\prime} M_{p}\left(I_{p}+r M_{p}\right)^{-2} \eta_{2} \leq 0\right\}
\end{aligned}
$$

Since the normal distribution is a spherically symmetric distribution (Ma \& Wang, 2009), by the properties of spherically symmetric distribution, $\psi_{1}=$ $\eta_{1}^{\prime}\left(I_{p}+r_{1} M_{p}\right)^{-2} M_{p} \eta_{1}$ and $R_{1}=\frac{1}{\left(\eta_{1}^{\prime}\left(I_{p}+r M_{p}\right)^{-2} M_{p} \eta_{1}\right)^{\frac{1}{2}}} \eta_{1}$ are independent. Similarly, wehave $\psi_{2}=\eta_{2}^{\prime}\left(I_{p}+r M_{p}\right)^{-2} M_{p} \eta_{2}$ and $R_{2}=\frac{1}{\left(\eta_{2}^{\prime}\left(I_{p}+r M_{p}\right)^{-2} M_{p} \eta_{2}\right)^{\frac{1}{2}}} \eta_{2}$ are independent. $\psi_{1}, \psi_{2}$ have the same distribution $R_{1}, R_{2}$ have the same distribution. 
Then, equation (21) can be reduced to:

$$
\begin{aligned}
& P_{r}\left\{-\psi_{1}+\left(\psi_{1} \psi_{2}\right)^{\frac{1}{2}} R_{1}^{\prime}\left(I_{p}+r M_{p}\right)^{-2} M_{p}^{\frac{1}{2}} R_{2}\right. \\
& \left.+\left(\psi_{1} \psi_{2}\right)^{\frac{1}{2}} R_{2}^{\prime}\left(I_{p}+r M_{p}\right)^{-2} M_{p}^{\frac{1}{2}} R_{1}+\psi_{2} \leq 0\right\}
\end{aligned}
$$

Since $R_{1}$ and $-R_{1}$ have the same distribution, $\psi_{1}$ is independent from $\psi_{2}$ and they also have the same distribution, we can change $R_{1}$ as $-R_{1}$ and change $\psi_{1}$ as $\psi_{2}$ in equation (22), then we obtain,

$$
\begin{aligned}
& P_{r}\left\{-\psi_{1}+\left(\psi_{1} \psi_{2}\right)^{\frac{1}{2}} R_{1}^{\prime}\left(I_{p}+r M_{p}\right)^{-2} M_{p}^{\frac{1}{2}} R_{2}\right. \\
& \left.+\left(\psi_{1} \psi_{2}\right)^{\frac{1}{2}} R_{2}^{\prime}\left(I_{p}+r M_{p}\right)^{-2} M_{p}^{\frac{1}{2}} R_{1}+\psi_{2} \leq 0\right\} \\
= & P_{r}\left\{-\psi_{2}-\left(\psi_{1} \psi_{2}\right)^{\frac{1}{2}} R_{1}^{\prime}\left(I_{p}+r M_{p}\right)^{-2} M_{p}^{\frac{1}{2}} R_{2}\right. \\
& \left.-\left(\psi_{1} \psi_{2}\right)^{\frac{1}{2}} R_{2}^{\prime}\left(I_{p}+r M_{p}\right)^{-2} M_{p}^{\frac{1}{2}} R_{1}+p s i_{1} \leq 0\right\} \\
= & 1-P_{r}\left\{-\psi_{2}-\left(\psi_{1} \psi_{2}\right)^{\frac{1}{2}} R_{1}^{\prime}\left(I_{p}+r M_{p}\right)^{-2} M_{p}^{\frac{1}{2}} R_{2}\right. \\
& \left.-\left(\psi_{1} \psi_{2}\right)^{\frac{1}{2}} R_{2}^{\prime}\left(I_{p}+r M_{p}\right)^{-2} M_{p}^{\frac{1}{2}} R_{1}+\psi_{1} \geq 0\right\} \\
= & 1-P_{r}\left\{-\psi_{1}+\left(\psi_{1} \psi_{2}\right)^{\frac{1}{2}} R_{1}^{\prime}\left(I_{p}+r M_{p}\right)^{-2} M_{p}^{\frac{1}{2}} R_{2}\right. \\
& \left.+\left(\psi_{1} \psi_{2}\right)^{\frac{1}{2}} R_{2}^{\prime}\left(I_{p}+r M_{p}\right)^{-2} M_{p}^{\frac{1}{2}} R_{1}+\psi_{2} \leq 0\right\}
\end{aligned}
$$

By equation (23), we obtain;

$$
\begin{aligned}
& P_{r}\left\{-\psi_{1}+\left(\psi_{1} \psi_{2}\right)^{\frac{1}{2}} R_{1}^{\prime}\left(I_{p}+r M_{p}\right)^{-2} M_{p}^{\frac{1}{2}} R_{2}\right. \\
& \left.+\left(\psi_{1} \psi_{2}\right)^{\frac{1}{2}} R_{2}^{\prime}\left(I_{p}+r M_{p}\right)^{-2} M_{p}^{\frac{1}{2}} R_{1}+\psi_{2} \leq 0\right\}=\frac{1}{2}
\end{aligned}
$$

Thus, we have,

$$
P C\left(\beta^{*}(r), \hat{\beta}, \beta\right) \geq \frac{1}{2}
$$

That is, when $\sigma_{i}, i=1,2$ are known and $\sigma_{2} \leq 2 \sigma_{1}$; the estimator $\beta^{*}(r)$ is superior over the estimator $\hat{\beta}$ under the $\mathrm{PC}$ criterion.

\section{Comparison of the estimator $\hat{\beta}$ and the estimator $\beta^{*}(r)$ under the $\mathbf{P C}$ criterion}

Now we compare the estimator $\tilde{\beta}$ given in equation (5) and the estimator $\beta^{*}(r)$ given in equation (7) under the PC criterion.

Theorem 2.2. when $\sigma_{1}, \sigma_{2}$ are known and $\sigma_{1} \leq 2 \sigma_{2}$, then the estimator $\beta^{*}(r)$ given in equation (7) is superior over the estimator $\tilde{\beta}$ given in equation (5) in the PC criterion.

Proof. Matrices $X_{1}^{\prime} Q_{1} X_{1}$ and $X_{2}^{\prime} Q_{2} X_{2}$ are positive definite and they can be diagonalised simultaneously, that is, there exists a reversible matrix $G$ such that,

$G^{\prime} X_{2}^{\prime} Q_{2} X_{2} G=I_{p}$

and

$G^{\prime} X_{1}^{\prime} Q_{1} X_{1} G=\operatorname{diag}\left(s_{1}, \ldots, s_{p}\right)=S_{p}$

with $s_{1}, \ldots, s_{p}$ showing the positive eigenvalues of matrix $X_{1}^{\prime} Q_{1} X_{1}\left(X_{2}^{\prime} Q_{2} X_{2}\right)^{-1}$. Define:

$G^{\prime} X_{2}^{\prime} Q_{2} \varepsilon_{2}=\zeta_{1}$

$\frac{1}{r} S_{p}^{-1 / 2} G^{\prime} X_{1}^{\prime} Q_{1} \varepsilon_{1}=\rho_{1}$

where $\zeta_{1}$ and $\rho_{1}$ present p-dimension column random vectors. It is easy to compute that $\zeta_{1} \sim N\left(0, \sigma_{2} I_{p}\right)$ and $\rho_{1} \sim N\left(0, \sigma_{2} I_{p}\right)$, and $\zeta_{1}$ is independent of $\rho_{1}$. Choose $U=X_{2}^{\prime} Q_{2} X_{2}$. As $Q_{i} Z_{i}=0, i=1,2$, by definition 2.1, we have,

$$
\begin{aligned}
L(\tilde{\beta}, \beta) & =(\tilde{\beta}-\beta)^{\prime} X_{2}^{\prime} Q_{2} X_{2}(\tilde{\beta}-\beta) \\
& =\left(\left(X_{2}^{\prime} Q_{2} X_{2}\right)^{-1} X_{2}^{\prime} Q_{2} y_{2}-\beta\right)^{\prime} X_{2}^{\prime} Q_{2} X_{2}\left(\left(X_{2}^{\prime} Q_{2} X_{2}\right)^{-1} X_{2}^{\prime} Q_{2} y_{2}-\beta\right) \\
& =\left(G^{\prime} X_{2}^{\prime} Q_{2} \varepsilon_{2}\right)^{\prime}\left(G^{\prime} X_{2}^{\prime} Q_{2} \varepsilon_{2}\right)=\zeta_{1}^{\prime} \zeta_{1}
\end{aligned}
$$

$$
\begin{aligned}
L\left(\beta^{*}(r), \beta\right)= & \left(\beta^{*}(r)-\beta\right)^{\prime} X_{2}^{\prime} Q_{2} X_{2}\left(\beta^{*}(r)-\beta\right) \\
= & \left(\left(X_{1}^{\prime} Q_{1} X_{1}+r X_{2}^{\prime} Q_{2} X_{2}\right)^{-1}\left(X_{1}^{\prime} Q_{1} y_{1}+r X_{2}^{\prime} Q_{2} y_{2}\right)-\beta\right)^{\prime} X_{2}^{\prime} Q_{2} X_{2} \times\left(\left(X_{1}^{\prime} Q_{1} X_{1}+r X_{2}^{\prime} Q_{2} X_{2}\right)^{-1}\right. \\
& \left.\left(X_{1}^{\prime} Q_{1} y_{1}+r X_{2}^{\prime} Q_{2} y_{2}\right)-\beta\right) \\
= & \left(\left(\frac{1}{r} X_{1}^{\prime} Q_{1} X_{1}+X_{2}^{\prime} Q_{2} X_{2}\right)^{-1}\left(X_{1}^{\prime} Q_{1} y_{1}+\frac{1}{r} X_{2}^{\prime} Q_{2} y_{2}\right)-\beta\right)^{\prime} X_{2}^{\prime} Q_{2} X_{2} \times\left(\left(\frac{1}{r} X_{1}^{\prime} Q_{1} X_{1}+X_{2}^{\prime} Q_{2} X_{2}\right)^{-1}\right. \\
& \left.\left(X_{1}^{\prime} Q_{1} y_{1}+\frac{1}{r} X_{2}^{\prime} Q_{2} y_{2}\right)-\beta\right) \\
= & \left(G^{\prime} X_{2}^{\prime} Q_{2} \varepsilon_{2}+\frac{1}{r} G^{\prime} X_{1}^{\prime} Q_{1} \varepsilon_{1}\right)^{\prime}\left(I_{p}+\frac{1}{r} M_{p}\right)^{-2} \times\left(G^{\prime} X_{2}^{\prime} Q_{2} \varepsilon_{2}+\frac{1}{r} G^{\prime} X_{1}^{\prime} Q_{1} \varepsilon_{1}\right)
\end{aligned}
$$




$$
\begin{aligned}
& =\left(\zeta_{1}+S_{p}^{1 / 2} \rho_{1}\right)^{\prime}\left(I_{p}+\frac{1}{r} S_{p}\right)^{-2}\left(\zeta_{1}+S_{p}^{1 / 2} \rho_{1}\right) \\
& =\zeta_{1}^{\prime}\left(I_{p}+\frac{1}{r} S_{p}\right)^{-2} \zeta_{1}+\zeta_{1}^{\prime} S_{p}^{1 / 2}\left(I_{p}+\frac{1}{r} S_{p}\right)^{-2} \rho_{1}+\rho_{1}^{\prime} S_{p}^{1 / 2}\left(I_{p}+\frac{1}{r} S_{p}\right)^{-2} \zeta_{1}+\rho_{1}^{\prime} S_{p}\left(I_{p}+r S_{p}\right)^{-2} \rho_{1}
\end{aligned}
$$

Thus, we have,

$$
\begin{aligned}
P C\left(\beta^{*}(r), \tilde{\beta}, \beta\right)= & P_{r}\left(L\left(\beta^{*}(r), \beta\right) \leq L(\tilde{\beta}, \beta)\right) \\
= & P_{r}\left\{\zeta_{1}^{\prime}\left(I_{p}+\frac{1}{r} S_{p}\right)^{-2} \zeta_{1}+\zeta_{1}^{\prime} S_{p}^{1 / 2}\left(I_{p}+\frac{1}{r} S_{p}\right)^{-2} \rho_{1}+\rho_{1}^{\prime} S_{p}^{1 / 2}\left(I_{p}+\frac{1}{r} S_{p}\right)^{-2} \zeta_{1}+\right. \\
& \left.\rho_{1}^{\prime} S_{p}\left(I_{p}+r S_{p}\right)^{-2} \rho_{1} \leq \zeta_{1}^{\prime} \zeta_{1}\right\} \\
= & P_{r}\left\{\zeta_{1}^{\prime}\left[\left(I_{p}+\frac{1}{r} S_{p}\right)^{-2}-I\right] \zeta_{1}+\zeta_{1}^{\prime} S_{p}^{1 / 2}\left(I_{p}+\frac{1}{r} S_{p}\right)^{-2} \rho_{1}+\rho_{1}^{\prime} S_{p}^{1 / 2}\left(I_{p}+\frac{1}{r} S_{p}\right)^{-2} \zeta_{1}+\right. \\
& \left.\rho_{1}^{\prime} S_{p}\left(I_{p}+r S_{p}\right)^{-2} \rho_{1} \leq 0\right\} \\
= & P_{r}\left\{\zeta_{1}^{\prime}\left(I_{p}+\frac{1}{r} S_{p}\right)^{-2}\left(-\frac{2}{r} S_{p}+\frac{1}{r^{2}} S_{p}^{2}\right) \zeta_{1}+\zeta_{1}^{\prime} S_{p}^{1 / 2}\left(I_{p}+\frac{1}{r} S_{p}\right)^{-2} \rho_{1}+\rho_{1}^{\prime} S_{p}^{1 / 2}\left(I_{p}+\frac{1}{r} S_{p}\right)^{-2} \zeta_{1}+\right. \\
& \left.\rho_{1}^{\prime} S_{p}\left(I_{p}+r S_{p}\right)^{-2} \rho_{1} \leq 0\right\} \\
\geq & P_{r}\left\{-\frac{2}{r} \zeta_{1}^{\prime} S_{p}\left(I_{p}+\frac{1}{r} S_{p}\right)^{-2} \zeta_{1}+\zeta_{1}^{\prime} S_{p}^{1 / 2}\left(I_{p}+\frac{1}{r} S_{p}\right)^{-2} \rho_{1}+\rho_{1}^{\prime} S_{p}^{1 / 2}\left(I_{p}+\frac{1}{r} S_{p}\right)^{-2} \zeta_{1}+\right. \\
& \left.\rho_{1}^{\prime} S_{p}\left(I_{p}+r S_{p}\right)^{-2} \rho_{1} \leq 0\right\}
\end{aligned}
$$

Since $\frac{2}{r} \geq 1$, then we $r \leq 2$, that is $\sigma_{1} \leq 2 \sigma_{2}$, and we obtain,

$$
\begin{aligned}
P C\left(\beta^{*}(r), \tilde{\beta}, \beta\right) \geq \quad & P_{r}\left\{-\zeta_{1}^{\prime} S_{p}\left(I_{p}+\frac{1}{r} S_{p}\right)^{-2} \zeta_{1}+\zeta_{1}^{\prime} S_{p}^{1 / 2}\left(I_{p}+\frac{1}{r} S_{p}\right)^{-2} \rho_{1}\right. \\
& \left.+\rho_{1}^{\prime} S_{p}^{1 / 2}\left(I_{p}+\frac{1}{r} S_{p}\right)^{-2} \zeta_{1}+\rho_{1}^{\prime} S_{p}\left(I_{p}+r S_{p}\right)^{-2} \rho_{1} \leq 0\right\}
\end{aligned}
$$

Then following the proof of Theorem 2.1, we get,

$$
P C\left(\beta^{*}(r), \tilde{\beta}, \beta\right) \geq \frac{1}{2}
$$

$\sigma_{1}, \sigma_{2}$ are known and $\sigma_{1} \leq 2 \sigma_{2}$, then the estimator $\beta^{*}(r)$ given in equation (7) is superior over the estimator $\tilde{\beta}$ given in equation (5) under the PC criterion.

\section{A SIMULATION STUDY}

In this section we present simulation results to validate the theoretical results. The models are given as follows:

$$
\begin{aligned}
& y_{1}=X_{1} \beta+Z_{1} \beta_{1}+\varepsilon_{1}, \varepsilon_{1} \sim N\left(0, \sigma_{1} I_{3}\right) \\
& y_{2}=X_{2} \beta+Z_{2} \beta_{2}+\varepsilon_{2}, \varepsilon_{2} \sim N\left(0, \sigma_{2} I_{3}\right)
\end{aligned}
$$

where

$$
X_{1}=\left(\begin{array}{ll}
1 & 2 \\
3 & 4 \\
5 & 6
\end{array}\right), Z_{1}=\left(\begin{array}{cc}
5 & 6 \\
7 & 8 \\
9 & 10
\end{array}\right), \beta=\left(\begin{array}{c}
\frac{\sqrt{3}}{3} \\
\frac{\sqrt{3}}{3} \\
\frac{\sqrt{3}}{3}
\end{array}\right)
$$$$
\beta_{1}=\left(\begin{array}{l}
1 \\
2 \\
3
\end{array}\right)
$$

$$
X_{2}=\left(\begin{array}{cc}
1 & 3 \\
5 & 7 \\
9 & 11
\end{array}\right), Z_{2}=\left(\begin{array}{ll}
10 & 18 \\
25 & 30 \\
40 & 60
\end{array}\right), \beta=\left(\begin{array}{c}
\frac{\sqrt{3}}{3} \\
\frac{\sqrt{3}}{3} \\
\frac{\sqrt{3}}{3}
\end{array}\right)
$$

$\beta_{2}=\left(\begin{array}{l}10 \\ 20 \\ 30\end{array}\right)$ 
For the values of $\sigma_{1}$ and $\sigma_{2}$, see the Tables 1and 2. Now we compute $P C\left(\beta^{*}(r), \hat{\beta}, \beta\right)$ and $P C\left(\beta^{*}(r), \tilde{\beta}, \beta\right)$. The simulation is replicated 10000 times.

By Table 1 , the estimator $\beta^{*}(r)$ is superior to the estimator $\hat{\beta}$ under the PC criterion. By Table 2, the estimator $\beta^{*}(r)$ is superior to the estimator $\tilde{\beta}$ under the PC criterion. By Tables 1 and 2, we find that our simulation results agree with the Theorems 2.1 and 2.2.

Table 1: The estimated $P C\left(\beta^{*}(r), \hat{\beta}, \beta\right)$ for $\sigma_{1}=0.1$ and different values $\sigma_{2}$

\begin{tabular}{llllll}
$\sigma_{2}$ & 0.01 & 0.02 & 0.05 & 0.1 & 0.2 \\
\hline$P C\left(\beta^{*}(r), \hat{\beta}, \beta\right)$ & 0.9684 & 0.9668 & 0.9686 & 0.9678 & 0.9648
\end{tabular}

Table 2: The estimated $P C\left(\beta^{*}(r), \tilde{\beta}, \beta\right)$ for $\sigma_{2}=0.1$ and different values $\sigma_{1}$

\begin{tabular}{llllll}
\hline$\sigma_{1}$ & 0.01 & 0.02 & 0.05 & 0.1 & 0.2 \\
\hline$P C\left(\beta^{*}(r), \tilde{\beta}, \beta\right)$ & 0.9328 & 0.9417 & 0.9536 & 0.9618 & 0.9672
\end{tabular}

\section{CONCLUSION}

In this article, we have compared the estimators in system $\mathrm{H}$ under the $\mathrm{PC}$ criterion. We have proven that the estimators in two linear equations in system $\mathrm{H}$ are superior over the estimators in a single equation system with known variances and under certain conditions. In future research, we will study the comparison among the estimators in system $\mathrm{H}$ under PC criterion when the variances are unknown.

\section{Acknowledgements}

This work was sponsored by the Natural Science Foundation of Chongqing (Grant No. cstc2019jcyjmsxmX0379) and the Scientific and Technological Research Program of Chongqing Municipal Education Commission (Grant No. KJQN201901347).

\section{REFERENCES}

Ahmadi J. \& Balakrishnan N. (2009). Pitman closeness of record values to population quantiles. Statistics and Probability Letters 79: 2037-2044.

DOI: https://doi.org/10.1016/j.spl.2009.06.016

Ahmadi J. \& Balakrishnan N. (2010). Pitman closeness of current records for location-scale families. Statistics and Probability Letters 80: 1577-1583.

DOI: https://doi.org/10.1016/j.spl.2010.06.010.

Jozani M.J. (2014). A note on Pitman's measure of closeness with balanced loss function. Statistics 48: 552-557. DOI: https://doi.org/10.1080/02331888.2012.751387

Keating J.P., Mason R.L. \& Sen P.K. (1993). Pitman Measure of Closeness: Comparison of Statistical Estimators. SIAM, Philadelphia, USA.

DOI: https://doi.org/10.1137/1.9781611971576

Liu A.Y. (1996). Estimation of the parameters in two linear models with only some of the parameter vectors identical. Statistics and Probability Letters 4: 369-350.

Li W.X., Yang H. \& Wu J.B. (2012). Some comments on: Ozkale M.R., Kaciranlar K. (2008): Comparisons of the r-k class estimator to the ordinary least squares estimator under the Pitman's closeness criterion. Stat Pap 53(2): 497-503.

Ma T.F. \& Wang S.G. (2009). Estimation of the parameters in a two linear regression equations system with identical parameter vectors. Statistics and Probability Letters 9: 1135-1140.

DOI: https://doi.org/10.1016/j.spl.2008.10.023

Pitman E.J.G. (1937). The closest estimates of statistical parameters. Mathematical Proceedings of the Cambridge Philosophical Society 33: 212-222.

DOI: https://doi.org/10.1017/S0305004100019563

Rao C.R. (1981). Some comments on the minimum mean square error as a criterion of estimation. In: Statistics and Related Topics, pp. 123-143. North Holland, The Netherlands. DOI: https://doi.org/10.21236/ADA093824

Reif J. (2006). Pitman closeness in classes of general pre-test and regression estimators. Communications in StatisticsTheory and Methods 35: 263-280.

DOI: https://doi.org/10.1080/03610920500440016

Wang S.G. \& Yang H. (1994). Linear estimators under pitman nearness criterion. Chinese Science Bulletin 40(23): 1944 1948.

Wu J.B. (2014). Comparison of some estimators under the Pitman's closeness criterion in linear regression model. Journal of Applied Mathematics 2014: Article ID 654949. DOI: https://doi.org/10.1155/2014/654949

Wu J.B. (2017). The small sample properties of the restricted principal component regression estimator in linear regression model. Communications in Statistics-Theory and Methods 46(4): 1661-1667.

DOI: https://doi.org/10.1080/03610926.2015.1024867

Yang H., Li W.X. \& Xu J.W. (2010). Comparison of two estimators of parameters under Pitman nearness criterion. Communications in Statistics-Theory and Methods 39: 3081-3094.

DOI: https://doi.org/10.1080/03610920903181985 